\title{
Mise en tension « des savoirs » et négociations identitaires
}

Exploration dans une université alternative

"Knowledge" tension-raising and identity negotiation. Exploration in an alternative university

\section{Sophie Del Fa}

\section{OpenEdition}

Journals

Édition électronique

URL : http://journals.openedition.org/communicationorganisation/5204

DOI : 10.4000/communicationorganisation. 5204

ISSN : $1775-3546$

\section{Éditeur}

Presses universitaires de Bordeaux

\section{Édition imprimée}

Date de publication : 1 juin 2016

ISBN : 979-10-300-0059-7

ISSN : 1168-5549

\section{Référence électronique}

Sophie Del Fa, « Mise en tension « des savoirs » et négociations identitaires », Communication et organisation [En ligne], 49 | 2016, mis en ligne le 01 juin 2019, consulté le 02 janvier 2020. URL : http:// journals.openedition.org/communicationorganisation/5204; DOI : 10.4000/ communicationorganisation.5204 


\title{
Mise en tension «des savoirs» et négociations identitaires Exploration dans une université alternative
}

\author{
Sophie Del fo ${ }^{1}$
}

\section{Introduction}

Cet article propose les résultats d'une ethnographie organisationnelle menée à l'ULibre, université alternative québécoise créée en 2010. Sa mission principale est de « favoriser le développement de l'esprit critique en offrant à la population un accès libre et gratuit au savoir par le biais d'activités d'éducation populaire implantées dans plusieurs quartiers de la ville $»^{2}$. Nous proposons d'explorer et d'ouvrir la discussion sur ce qu'est une organisation alternative et surtout la façon dont ses membres construisent et font émerger une identité à partir de la négociation de différents types de savoir.

Les universités populaires (UP) sont nées en France en octobre 1899 en pleine clôture de l'affaire Dreyfus. Georges Deherme, ouvrier typographe, voulait alors créer des rencontres libres et gratuites entre intellectuels républicains et ouvriers. Dans le sillage des Lumières, il soutenait une éducation fondée sur la raison critique, l'émancipation, l'autonomisation individuelle, les solidarités non oppressives et polyphoniques afin de développer des repères critiques. Ces universités sont dites "populaires » au sens le plus noble du terme : elles concernent et appartiennent au peuple. Disparues dès les débuts de la Première Guerre mondiale faute d'implication et à cause d'un contenu trop théorique, les universités populaires réapparaissent dans les années 1960 et éclosent dans plusieurs pays d'Europe et d'Amérique du Nord. Aujourd'hui, les postulats des UP sont triples: 1) proposer une pluralité de points de vue argumentés et rigoureux, 2) encourager les débats critiques afin de participer à l'activité intellectuelle, 3) assumer que les interrogations philosophiques ou les connaissances des sciences sociales relèvent davantage d'un processus que de données assénées (Corcuff 2008 : 2). Le projet repose sur la liberté

1 Sophie Del Fa est Doctorante en communication à l’Université du Québec à Montréal ; sophie.delfa@gmail. com

2 Éléments recueillis sur le site internet de l’ULibre. 
inconditionnelle de questionnement et de proposition, sur le bénévolat et le plaisir réciproque entre enseignants/enseignés, sur la mobilité intellectuelle, la souplesse pédagogique, l'imagination et sur des savoirs critiques militants (Corcuff 2008).

L'histoire et la définition des UP leur confèrent une place unique dans le paysage de l'éducation. Dans le cadre de cette analyse, nous nous intéressons à la façon dont elles construisent et négocient leur identité. De fait, en tant qu'organisations alternatives, elles sont considérées comme des organisations fragiles qui se doivent d'effectuer un travail important afin de figer et d'asseoir clairement ce qu'elles sont. En tant qu'organisme d'éducation, l'identité s'articule autour de la notion de savoir sur laquelle nous focalisons notre attention.

Les observations menées à l'ULibre ont dévoilé la présence en tension de plusieurs types de savoir : 1) un savoir libre et gratuit, propre à l'idée d'université telle que définie notamment par les idéalistes allemands (voir à ce propos, entre autre, Habermas 1987), 2) le rejet absolu d'un savoir marchandisé et 3) une savoir militant ayant pour objectif d'encourager l'action citoyenne risquant alors une assimilation de l'ULibre à des groupes politiques activistes. Nous nous sommes demandé comment cette tension, mise en acte par les membres lors de réunions et pendant les cours, agit sur l'identité de lorganisme. Nous nous intéressons à la façon dont la définition du contenu du savoir renouvelle, mais aussi et surtout fragilise l'identité de l'organisation.

Nous proposons tout d'abord une revue de la littérature sur les notions d'identité, de tensions et de savoir. Avant le développement de l'analyse du contenu d'une réunion du comité, nous présenterons l'approche constitutive de la communication à partir de laquelle nous concevons l'identité organisationnelle comme étant co-construite à travers des processus continus de communication.

\section{Identité, tensions et savoir à l'ULibre: une approche communica- tionnelle}

Identité et tension: difficile définition des organisations alternatives

De l'identité organisationnelle comme élément instable et négocié

Deux traditions analytiques ont marqué la recherche sur l'identité organisationnelle: l'approche essentialiste et l'approche anti-essentialiste (Hatch et Schultz 1997; Ran et Golden 2011; Sveningsson et Alvesson 2003). C'est sur cette deuxième approche que notre conception de l'identité repose. En effet, suivant les arguments de Hatch et Shultz (1997), nous postulons que l'identité organisationnelle renvoie à ce que les membres perçoivent, ressentent et pensent vis-à-vis de l'organisation dans laquelle ils évoluent. Aussi, nous admettons que l'identité organisationnelle est collective et surtout 
que la compréhension des valeurs et des caractéristiques de l'organisation est partagée parmi les membres (librement traduit de Hatch et Schultz 1997:357). Hatch et Schultz décomposent l'identité organisationnelle en trois éléments interreliés : l'identité (relative aux membres), l'image (relative aux non-membres) et la culture (relative à l'histoire et aux valeurs). De fait, nous ne pouvons pas séparer l'identité, l'image et la culture. Ran et Golden, de leur côté, estiment que l'identité organisationnelle est intentionnelle, temporelle, relationnelle et externe puisquelle interagit et se transforme en fonction des changements de l'environnement (2011). Elle est intentionnelle parce qu'elle est inscrite dans une «stratégie politique» et est induite par certaines motivations; temporelle parce quelle est construite comme un résultat de négociations à partir de certains événements qui se sont déroulés à un moment donné précis du temps; relationnelle parce qu'elle est liée à son dehors, c'est-à-dire aux différentes parties prenantes, aux changements environnementaux et au champ institutionnel dans lequel elle s'inscrit ; enfin, elle est toujours orientée vers l'externe parce qu'elle est toujours partagée à l'extérieur de l'organisation à travers les échanges avec son «audience».

En somme, l'identité organisationnelle répond à la question "Qui suisje?» (Sveningsson \& Alvesson 2003 :1168) et s'y attarder suppose d'explorer la façon dont les membres font sens de ce qu'est -ou de ce que doit êtrelorganisation pour eux et pour les autres (c'est-à-dire les personnes qui ne font pas partie de l'organisation).

Par ailleurs, l'identité n'est pas fixe, mais elle est mouvante, instable, sans cesse redéfinie et renégociée (Ran \& Golden 2011:418). Cette dimension en mouvement de l'identité nous intéresse tout particulièrement puisque, comme les analyses le montreront, nous sommes en présence d'une organisation qui négocie et qui redéfinit son identité. Nous lions cette dynamique à la notion de tension que nous considérons comme étant constitutive des organisations à l'étude et surtout à une difficile définition de ce qu'est l'alternatif.

\section{Organisations alternatives : tensions et fragilité}

Un large pan de la littérature sur les organisations s'accorde pour les définir comme étant mues par des tensions (Eikenberry 2009; Sanders 2012; Trethewey \&Ashcraft 2013). Selon Trethewey et Ashcraft (2013), ces tensions ne sont pas des anomalies, mais plutôt des « irrationalités " symptomatiques d'un état normal et qui caractérisent une "vie organisationnelle ». Les deux auteures rappellent en effet que les organisations sont avant tout des «sites de conflits » et de plus, elles ajoutent que lorsqu'une organisation tend à s'organiser " différemment ", elle est inévitablement mue par des contradictions entre des idéologies, des structures et des pratiques différentes. Ce dernier point nous intéresse particulièrement puisque l'ULibre est une université alternative qui justement s'organise différemment. Ainsi, les tensions ici sont d'autant plus 
centrales et nous postulons qu'elles agiront sur la manière dont elle se définit et qu'elles mettent à mal la gestion de son identité. Mais qu'est-ce qu'une organisation alternative au-delà de ces tensions?

Parker, Cheney, Fournier et Land soulignent que ce n'est pas toujours facile de décider de ce qui est " alternatif » (2014:34, notre traduction) parce que les organisations alternatives ne vont pas de soi. De prime abord, elles représentent un ensemble de valeurs, de principes voire de positionnements idéologiques. Selon, Parker, Cheney, Fournier et Land, leurs deux caractéristiques principales sont leurs aspects contestataire et politique (2014). Dans les faits, une organisation alternative repose sur une volonté d'améliorer l'ordre social avec des idées utopiques (Parker, Fournier, et Reedy 2007; Parker 1998) afin de bousculer ce qui est conventionnel. Par ailleurs, les alternatives sont nombreuses et elles ne sont pas toujours éthiquement acceptables ; par exemple l'esclavagisme, le fascisme, le féodalisme sont des alternatives, mais elles n'améliorent pas l'ordre social. Il est donc peu aisé de saisir ce que sont ces organisations. Par le fait même, nous postulons qu'il est difficile pour une organisation comme telle de se définir elle-même ; les tensions et contradictions sont alors des enjeux à manipuler avec précaution. Dans cette perspective, nous explorons l'ULibre en tant qu'organisation alternative afin de comprendre l'aspect négocié de l'identité, mais aussi pour participer à la discussion autour de la question : qu'est-ce que c'est qu'une organisation alternative ${ }^{3}$ ?

Ainsi, nous proposons de comprendre plus précisément de quoi sont faites les tensions qui parcourent l'ULibre en tant qu'université alternative dont nous avons du mal à saisir la teneur. En tant qu'université, nous proposons une réflexion à partir de la notion de savoir comme élément structurant. Là encore nous ouvrons sur un terme qui est loin d'être univoque.

\section{Savoir pur, savoir marchand et savoir activiste}

Le principe fondamental des universités est historiquement construit autour de l'idée que l'université propose un savoir académique éclairé et libre (Macherey 2011) et donc « vrai ». À ce propos, Derrida écrit que l'université fait une "profession de foi » $(2001: 23)$ envers le savoir. Originalement, ce savoir théorique engageait les membres de l'université vers « le haut». Cependant, comme le souligne Lyotard, la nature du savoir a changé au fil du temps et aujourd'hui, il fait partie intégrante de nos sociétés. De plus et surtout, il est « et sera produit pour être vendu, et il est et sera consommé pour être valorisé dans une nouvelle production : dans les deux cas, pour être échangé » (1979 : 6). Dans cette optique, le savoir est devenu un enjeu majeur et Lyotard en parle en termes de " mercantilisation ». Le savoir n'est plus neutre, libre

3 Cette interrogation nous vient de Cheney qui se demande : «What then do alternative organizations looks like?» (2014, n.p) 
et tourné vers la Vérité, mais il est « à vendre » et possède une valeur d'usage. Cela a un impact sur le savoir divulgué à l'université ; plusieurs académiciens ont soulevé justement que le savoir a désormais une valeur marchande en tant qu'il est la clé vers des emplois et par conséquent vers une meilleure situation économique (Alvesson 2013 ; Naidoo, Shankar, \& Veer 2011). Comme le souligne Derrida, en fait le savoir relève davantage du " métier ", de la " compétence »/savoir-faire (2001:35). La «Vérité » a disparu au profit d'une application pratique. Dans ce sillage, l'ULibre refuse justement la définition de ce savoir marchandisé et veut renouer avec un "savoir pur ", libre et désintéressé. Cependant, les universités populaires sont nées dans un contexte politique de contestations et ont été créées pour venir à bout d'inégalités induites par les universités traditionnelles; de fait ayant disparu pendant les deux Guerres Mondiales, elles se sont reformées progressivement à partir de mai 68 où la contestation étudiante contre la structure universitaire était à son maximum. De même, les universités populaires continuent d'émerger "contre» l'ordre établi, le capitalisme et la tour d'ivoire qu'est l'université. Ainsi, elles sont donc «chargées» politiquement et idéologiquement.

À l'ULibre, par exemple, les membres du comité sont engagés et ils se définissent comme étant « anarchistes» « de gauche» et «contrele capitalisme» ${ }^{4}$. Ils encouragent alors un savoir politisé qui mène à l'action citoyenne en vue de faire changer les choses. Les universités populaires sont à la croisée de trois types de savoirs qui induisent des identités différentes: un savoir pur, idéal, gratuit et libre qui tend vers l'émancipation et la connaissance universelle, un savoir marchandisé à forte valeur économique qui mène à l'obtention d'un métier (celui-ci est fortement rejeté en filigrane par l'ULibre) et un savoir tourné vers l'action chargé idéologiquement qui relève dans une certaine mesure de l'activisme. Ainsi, bien que ces trois types de savoir parcourent les universités, il est question ici de la tension entre le savoir libre et désintéressé et le savoir politisé.

\section{Approche constitutive de la communication et ventriloquie}

Notre réflexion se place dans une approche constitutive où la communication est conçue comme «un processus par lequel les choses, les animaux et les individus viennent à s'exprimer à travers une multitude d'incarnations et de matérialisations» (Cooren 2015; 2, notre traduction). Dans cette perspective, il n'y a pas de distinction entre le matériel (roches, tables, minéraux, atomes, et ordinateurs) et l'immatériel (le monde des esprits, les symboles, les émotions, les fantômes, les discours, les interactions) ${ }^{5}$. Autrement dit, toutes choses existent, se matérialisent, s'incarnent de différentes façons au cours de leur existence, mais toutes ces choses sont matérielles même si elles sont

4 Expressions tirées dans entrevues réalisées avec les membres de l'organisation.

5 Les termes entre parenthèses sont repris et traduis de Cooren 2015, p. 9. 
intangibles. Leur degré d'existence et leur mode varient et existent sous des formes différentes. Cooren résume cela en ces termes :

Materialization is thus directly related to questions of existence to the extent that beings come to exist more or less through what substantiates/materializes/mediatizes them. These materials are the means by which these beings literally pass through or come across this presentation (Cooren 2015).

C'est cette démarche-là que nous privilégions en tenant compte des principes proposés par Cooren (2015: 21-214). Selon lui, nous ne devons pas réduire les études en communication à des questions de construction sociale parce qu'en fait, tout être humain et non humain peut être perçu comme un acteur et un passeur. Dans cette relationnalité, toutes conversations, toutes présentations orales ou tout acte d'écriture sont des activités de médiatisation, cest-à-dire des activités à travers lesquelles un plénum d'êtres peut (par proxy ou à distance) s'exprimer et se révéler. Ainsi, selon Cooren : « la communication est constitutive du mode d'existence de tout être, que ce soit un être humain, une organisation, une ouvre d'art, une idée, une émotion, un virus ou une technologie » $(2015: 23)$. Cette constitution n'est pas un acte de réduction, mais elle consiste en la reconnaissance des dimensions relationnelles de toutes personnes et de toutes choses. Ainsi, la communication est la façon par laquelle tout être existe $\left(2015: 23\right.$, nous traduisons $\left.{ }^{6}\right)$.

Suivant cette approche, les tensions et les savoirs dont nous parlions sont des êtres qui font partie de la constitution de l'ULibre, ils sont en elle et ils la meuvent. Mais comment le font-elles et comment cela se répercute-t-il sur l'identité de l'organisation ? La construction identitaire des organisations est donc un enjeu de taille afin qu'elles se positionnent dans le paysage organisationnel. En ce sens, une approche constitutive permet de comprendre comment l'identité d'une organisation alternative émerge et quels éléments sont négociés pour construire cette identité.

Afin de proposer des pistes de réponses à ces interrogations, nous proposons une analyse ventriloque des conversations (Cooren 2010). La ventriloquie permet de suivre des figures que nous faisons parler et qui parlent dans les interactions. De plus, elle nous oblige à porter notre attention aux dimensions émotionnelles et passionnelles des interactions : invoquer telle figure ne démontre pas seulement une volonté d'affirmer dur comme fer un énoncé, mais dévoile un certain attachement à cette figure particulière, nous sommes liés d'une certaine manière à elle (Cooren 2013 : 264). Ainsi, lorsque les individus donnent un compte rendu de la réalité organisationnelle, ils introduisent l'organisation dans le dialogue par le biais de nombreux agents, humains ou non humains : cela peut être des cartables, des procédures, des règlements, des collègues qui sont invoqués dans le dialogue et qui

6 Phrase originale : «Communication is the way by which all these beings manage to exist, more or less» (2015 : 23) 
vont faire des choses. Les individus attribuent donc à d'autres leur propre agentivité, légitimant par le fait même leurs actions (Cooren 2006, 2010). Par la mobilisation d'une quantité d'entités dans le dialogue, l'organisation est animée par la ventriloquie, processus d'incarnation qui rend une entité présente dans la communication. En ce sens, nous mobilisons les figures qui deviennent des participantes «à part entière» des interactions et qui vont participer à la construction de l'identité.

Dans notre analyse, c'est moins la dynamique interactionnelle qui nous intéresse que le contenu des échanges entre les participants. De fait, nous focalisons notre attention sur ce qu'il s'est dit au cours d'une réunion du comité de l'ULibre pour comprendre comment ils font émerger et négocient l'identité de l'organisme et comment ces derniers exemplifient la difficulté de se définir en tant qu'université populaire alternative. Ainsi, nous proposons de suivre les trois types de savoir mis en avant précédemment pour comprendre comment leur mise en tension fait émerger des identités multiples voire contradictoires.

Les analyses sont issues d'un travail de terrain réalisé de janvier à avril 2015 suivant une méthode d'ethnographie organisationnelle (Neyland 2008; Van Maanen 2011; Ybemas, Yanow, Wels et Kamsteeg 2009). Nous avons effectué une observation non-participante d'une réunion-brunch de trois heures des membres du comité (22 février 2015) et assisté à quatre cours de la session d'hiver. Nous avons également conduit quatre entrevues semi-dirigées avec les membres du comité et observé la soirée de lancement de la session d'hiver 2015 qui a eu lieu le 5 février. Chaque observation a été complétée par la prise de notes de terrain.

\section{Analyse: des savoirs en tension et une identité négociée}

Plusieurs tours de parole de la réunion du comité exemplifient la mise en tension des types de savoir mentionnés ci-haut : libre, marchandisé et militant. Attardons-nous sur une séquence particulière à la fin de la réunion du comité observée où Lionel propose de discuter du futur de l'ULibre. Il est important ici d'indiquer que le comité en place au moment de l'étude est constitué de plusieurs nouveaux venus et, pour la plupart d'entre eux, c'est leur première réunion. Pour cette raison, Lionel tient à conclure sur l'avenir de l'ULibre pour s'assurer que chacun s'aligne vers des objectifs communs. Il propose d'introduire dans l'organisme un engagement clair envers l'action citoyenne. Cette proposition ne fait pas l'unanimité parmi les membres, certains ayant peur de voir l'ULibre se transformer en parti politique ou en organisme militant et donc de fragiliser voire de déplacer l'identité de l'organisation. Les membres doivent alors trouver un moyen de faire de l'action sociale sans pour autant transformer l'organisme en un parti politique. La réunion devient un «brainstorm » dans le but de trouver une solution pour préserver l'identité de 
l'organisme et se structure autour d'une négociation entre ceux qui défendent le militantisme et ceux qui sont plutôt du côté d'un savoir libre et désintéressé.

L'ULibre ne veut pas se transformer en parti politique parce qu'elle ne doit «pas être responsable» des actions qui en découleraient. Cela ne fait pas partie de ses valeurs. Pourtant Lionel lit le dépliant qui présente l'ULibre pour montrer que, bien qu'elle ne soit pas un parti politique, «l'action citoyenne» demeure une de ses vocations:

Lionel : ((il prend le dépliant et lit)) « Favoriser le développement de l'esprit critique ainsi que l'action citoyenne »

\section{((Silence))}

Lionel : C'est ça qui m’a starté quand j'ai vu ça j’ai fait « ah oui on a oublié de parler de ça...» Puis il ne faut pas dire aux gens d'aller dans tel groupe [politique]... mais juste de leur indiquer que les personnes peuvent faire quelque chose et dire 'voici le genre d'approche avec laquelle vous pouvez faire quelque chose' et lier le tout à des organismes... Je pense que cela est super pertinent. Mais il ne faut pas qu'il se dise ensuite « l'ULibre m'a dit de faire ça ». Il faut que cette prise d'action là reste dans une idée d'esprit critique. L'ULibre pourrait être un accompagnement énorme pour les profs pour bâtir cela... avec des outils rapides.

Source: réunion du comité 22 février 2015

Le dépliant est un actant non humain qui agit sur la définition de l'organisation. À partir de l'entrée en scène du dépliant, chaque membre se repositionne quant à l'identité de l'ULibre tantôt la définissant comme un organisme où le savoir doit être libre, tantôt comme une organisation où le savoir doit être utilisé en vue de changer les choses donc vers quelque chose qui s'apparente davantage à de l'activisme. Cooren (2010) dirait que Lionel fait parler le dépliant qui incarne l'ULibre. Ainsi, puisque le dépliant mentionne que l'action citoyenne fait partie des valeurs, il faut que cela se retrouve en pratique dans les cours. Deux principales craintes sont alors mises en avant par rapport à cette idée d'action citoyenne : tout d'abord, cela pourrait être un frein à la liberté et à l'esprit critique ; autrement dit, certains ont peur de voir le savoir libre et éclairé se transformer en un prosélytisme qui ne ferait que remplacer une idéologie par une autre :

Nina: [il faut] proposer un espace où les gens peuvent discuter entre eux, mais sans diriger vers des types d'actions spécifiques

Emma : [Oui évitons le prosélytisme...

Source: réunion du comité 22 février 2015

Et ensuite, la peur de rendre l'ULibre responsable des actions entreprises qu'elle ne pourrait pas contrôler: 
Edouard. : C'est vrai... moi j'ai le souvenir d'une affaire qu'on a faite à moment donné : ça avait été proposé par les organisateurs-trices, je me souviens plus quel était le cours, c'était peut-être un cours sur le féminisme, je ne sais plus lequel... il y'avait un nombre de sessions à l'ULibre puis y'avait une autre session, une autre séance en fait... une extraséance, donc l'ULibre était pas responsable c'est-à-dire que c'était les personnes externes qui l'organisaient.

Edouard : Il faut bien se positionner là-dedans étant donné qu'on n'est pas responsable, il ne faut pas que l'ULibre devienne responsable des actions prises.

Source: réunion du comité 22 février 2015

Ici, c'est l'expérience passée que Edouard fait parler. Les membres ne veulent pas que l'ULibre soit assimilée à des discours ou des actions qui peuvent lui échapper. En fait, les membres du comité craignent que certaines actions citoyennes ternissent l'identité de l'organisme en la transformant en groupe militant:

Nina: Peut-être que si on avait une structure plus réglo à ce niveau-là: on dit au prof que le dernier cours de la session c'est un cours « action citoyenne » qui est optionnel. Vous le donnez ou pas... puis, c'est là que les gens prennent la parole... (...) Moi ce dont j'ai peur c'est que tout le long du cours il y ait une espèce d'appel au militantisme sans arrêt (...) je veux avoir une conférence.

Nina: Parce qu'il y a des profs qui ne sont pas militants du tout et qui sont juste chercheurs.

Edouard: Il y a des profs qui ne sont pas militants, puis je pense qu'il faut garder ça flexible.

Source: réunion du comité 22 février 2015

L'ULibre est dépendante de ce qui se dit et se fait au sein de ses cours et elle ne peut pas se permettre de mettre en jeu sa position dans le paysage de l'éducation populaire. Nous avons remarqué la réticence notamment d'Édouard et de Nina envers l'appel à l'action citoyenne. Aussi, Élö̈se nous dira en entrevue: «je crois que l'ULibre n'a pas à faire de l'action citoyenne concrète ou organisée, pour nous ça ne se joue pas là, je ne trouverai pas ça intéressant».

Cependant, certains sont attachés à cette dimension:

Edouard : mais moi je serai assez ouvert, mais je ne sais pas comment le mettre [en place], peut-être à la fin du cours ou... dire que dans la « $3 \mathrm{e}$ heure » si certains veulent connaître les noms d'organismes subversifs pour poser des bombes ils peuvent rester... ((rires))

Source: réunion du comité 22 février 2015 
Bien que chaque membre du comité soit engagé pour des causes sociales et politiques, le terme de militantisme est diabolisé, car il va à l'encontre de la liberté qui supplante toutes les autres valeurs. Ainsi, au fur et à mesure que la tension se dénoue, Édouard propose d'avoir recours à un tiers pour engager l'action citoyenne:

Edouard : On ne peut pas forcer les profs à ça, mais on peut par le formulaire, par la manière d'aborder les professeurs leur suggérer cette dimension-là pour qu'ils y pensent ; et pour que ceux qui veulent apportent cette dimension... ((acquiescement)) dans leur cours. Dans le formulaire il faut qu'apparaisse la possibilité d'une séance supplémentaire où on s'organise et on passe à l'action. Le professeur n'est pas obligé de s’impliquer à ça...

Edouard : Mais même moi je dirais que l'ULibre nest même pas obligée d'organiser cela. Comme ce n'est pas l'ULibre qui décide qu'un professeur fait 4 séances ou 5 séances ou qu'il va parler d'une chose ou d'une autre, je pense que ce n'est pas l'ULibre qui doit décider qui a ça dans un cours.

Edouard : Et le prof après il dit : « voici ma proposition j’ai 5 séances puis après ma séance d'action citoyenne ou de prise de [position], je sais pas comment on va appeler ça » nous on dira qu'il y a 6 séances.

Source: réunion du comité 22 février 2015

Autrement dit, cette séance « d'action citoyenne » ne doit pas être initiée officiellement par l'ULibre, mais par les professeurs à travers le formulaire qu'ils ont à remplir lorsqu'ils proposent un cours via le site Internet. Le professeur ne fait pas à proprement parler partie de l'ULibre et peut donc faire office d'initiateur d'action citoyenne : il le ferait en son nom sans compromettre l'identité de l'université. Il est intéressant ici de voir comment le recours à un tiers permet de se délaisser de la responsabilité. Les professeurs sont ici mobilisés pour que le savoir reste en apparence libre et désintéressé mais puisse éventuellement se transformer en action. L'ULibre devient en filigrane une «facilitatrice » de l'action citoyenne. D'une certaine manière, elle instrumentalise les professeurs pour inciter une action dont elle ne peut pas être officiellement porteuse.

La négociation relative à ce que l'ULibre "peut être » et « ne peut pas être » découle aussi d'une place ambiguë dans le paysage des OBNL. Comme le dira Lionel lors de son entrevue :

On a le défaut de ne pas être dans aucune case, on ne s'occupe pas de femmes battues, on ne s'occupe pas de gens qui meurent de faim et cela est très important, mais on est pas un organisme caritatif au sens classique, on fait pas de léducation populaire aux analphabètes donc l'impact réel de l'ULibre est moins mesurable et est probablement moins grand à court terme que ces organismes-là.

Source: entrevue 11 mars 2015 
Le fait de « nêtre dans aucune case » est à la fois dû à la mission inhérente de l'ULibre, mais peut s'expliquer aussi par la tension mise en évidence entre savoir libre et savoir engagé ; l'ULibre est un organisme idéologique qui ne se définit pas par son statut d'OBNL donc d'organisation caritative, mais plutôt par des valeurs. De fait, alors que l'ULibre refuse l'idéologie, puisqu'elle ne veut pas être ouvertement politisée, elle est paradoxalement profondément idéologique puisqu'elle est mue par la tension explicitée jusqu'ici et surtout puisqu'elle se définit par des valeurs idéologiques. Dans le cas de l'ULibre, l'idéologie définit l'organisation. À l'image des organisations féministes (voir D'Enbeau et Buzzanell 2013) ou encore des organisations spirituelles (voir Molloy et Heath 2014), l'ULibre se définit d'abord et avant tout par un ensemble de valeurs qui contraignent les membres qui doivent s'atteler à trouver les moyens pour les contourner et pour ne pas être responsables de ce qui pourrait en découler. Ainsi, l'identité de l'ULibre repose sur sa relation à la responsabilité. Si nous relions cela à notre réflexion plus large sur les organisations alternatives, nous pourrions dire que leurs identités reposent donc en grande partie sur leur responsabilité quant aux actions quelles peuvent induire. Dans le cadre de cette université, la responsabilité se focalise sur la teneur du savoir divulgué.

\section{Conclusion}

Cette analyse nous a permis d'explorer en quoi plusieurs types de savoir sont en tension au sein de l'ULibre rendant difficile sa définition en tant qu'université alternative. En ce sens, l'identité est fragile et précaire parce qu'instable et mue par des contradictions qui ne la font entrer dans « aucune case ». Bien qu'alternative, cette organisation est à la recherche d'une identité qui puisse la définir. Se focaliser sur les différents types de savoir est pertinent dans le cadre d'une étude sur une université parce que c'est à partir de lui que l'on saisit la façon dont elle se définit. Ainsi, pour mieux comprendre ce qu'est une organisation alternative, il faut suivre ce qui la constitue et donc ce qui la définit. Par ailleurs, force est de constater qu'une organisation alternative est toujours à la croisée de plusieurs éléments qui fragilisent par le fait même ce qu'elles sont. À quoi ressemblent alors les organisations alternatives? Pour l'instant à un ensemble de valeurs et d'idéologies fragmentées et contradictoires. Au-delà d'une exploration des organisations alternatives, cet article a également permis de mettre en évidence la façon dont le savoir, dans sa définition la plus large, parcourt une organisation et constitue la pierre angulaire autour de laquelle émerge l'identité organisationnelle. 


\section{BIBLIOGRAPHIE}

ALVESSON M. (2013), The Triumph of Emptiness: consumption, higher education $\mathcal{E}^{\circ}$ work organization. UK: Oxford University Press.

CHENEY G. (2014), Alternative organization and alternative organizing.

COOREN F. (2010), Action and agency in dialogue: Passion, incarnation, and ventriloquism (John Benja). Amsterdam.

COOREN F. (2013), Manières de faire parler: Interaction et ventriloquie. Bordeaux, France: Le Bord de l'eau.

COOREN F. (2015), in Media Res : Rethinking Communication, Materiality and Existence from a Relational Approach. In ANZCA 2015 Rethinking Communication, Space and Identity (p. 1-26). Queenstown, NZ.

CORCUFF P. (2008), Universités populaires alternatives en France: un autre modèle est-il possible ? À Babord, (26), 2-5.

D’ENBEAU S. \&BUZZANELL P.M.(2013), Constructing a feminist organization's identity in a competitive marketplace: The intersection of ideology, image, and culture. Human Relations, 66(11), 1447-1470.

DERRIDA J. (2001), L'université sans condition. Galilée.

EIKENBERRY A. M. (2009), Refusing the Market. Nonprofit and Voluntary Sector Quarterly, 38(4), $582-596$.

HABERMAS, J. (1987), The Idea of the University - Learning Processes. New German Critique, (41), 3-22.

HATCH M. J. \& SCHULTZ M. (1997), Relations between organizational culture, identity and image. European Journal of Marketing, 31(5), 356-365.

MACHEREY P. (2011), La Parole universitaire. La Fabrique.

MOLLOY K. A. \& HEATH R. G. (2014), Bridge Discourses and Organizational Ideologies: Managing Spiritual and Secular Communication in a Faith-Based, Nonprofit Organization. International Journal of Business Communication, 51(4), 386-408.

NAIDOO R., SHANKAR A., \& VEER E. (2011), The consumerist turn in higher education : Policy aspirations and outcomes. Journal of Marketing Management, 27(October), 1142-1162.

NEWMAN J. H. (n.d.), L'idée d'université. (É. A. Solem, Ed.). London: Broché.

NEYLAND D. (2008), Organizational ethnography. Thousand Oaks, CA: Sage.

PARKER Martin, FOURNIER Valérie, REEDY P. (2007), The Dictionary of Alternatives : Utopianism and Organization (1st ed.). Zed Books.

PARKER M. (1998), Organisation, community and utopia. Studies in Cultures, Organizations and Societies, 4(1), 71-91.

PARKER M., CHENEY G., FOURNIER V. \& LAND C. (2014), The Routledge companion ot alternative organizing. Routledge.

RAN B. \& GOLDEN T. J. (2011), Who Are We?: The Social Construction of Organizational Identity Through Sense-Exchanging. Administration E Society, 43(4), 417-445. 
SANDERS M. L. (2012), Theorizing Nonprofit Organizations as Contradictory Enterprises: Understanding the Inherent Tensions of Nonprofit Marketization. Management Communication Quarterly, 26(1), 179-185.

SVENINGSSON S. \& ALVESSON M. (2003), Managing managerial identities : Organizational fragmentation, discourse and identity struggle. Human Relations, 56(10), 1163-1193.

TRETHEWEY A. \& ASHCRAFT K. L. (2013), Special Issue Introduction. Journal of Real Estate Finance and Economics, 46(4), 565-567.

VAN MAANEN J. (2011), Tales of the field: On writing ethnography. The University of Chicago Press.

YBEMAS S., YANOW D., WELS H., \& KAMSTEEG F. (2009), Studying everyday organizational life. Thousand Oaks, CA: Sage.

Résumé : Cet article propose les résultats d'une ethnographie organisationnelle réalisée dans une université alternative (l'ULibre). Nous focalisons notre attention sur plusieurs types de «savoir » (libre, marchandisé et tourné vers l'action politique), négociés entre les membres de l'organisation et agissant sur son identité afin de comprendre à quoi peut ressembler une organisation alternative et surtout comment elle construit son identité. Suivant une approche constitutive de la communication (CCO) et une analyse ventriloque du contenu des interactions, nous postulons que le savoir multiple et disparate fragilise l'identité de l'organisation. Nous questionnons la négociation identitaire d'une université populaire dite «alternative» afin d'explorer en bout de ligne les caractéristiques et la façon dont existe ce genre d'organisations.

Mots-clés : Organisations alternatives, tensions, identité organisationnelle, approche constitutive de la communication (CCO), ventriloquie.

\begin{abstract}
This article proposes the results of an organizational ethnography held in folk high school labelled as an "alternative university" (ULibre). Our focus is centered on three types of "knowledge" (free, marchandized and activist). We think that these three types of knowledge are negociated between the members of this organization and thus act upon its identity. Following a constitutive approach of communication (CCO) and a ventriloquist analysis of the interactions content we postulate through a multiple and disparate knowledge, the organization identity is weakened. We are questioning the ways the member's negotiate a folk high school identity in order to explore in fine the main characteristics and the mode of existence of alternative organizations.
\end{abstract}

Keywords: Alternative organizations, tensions, organizational identity, constitutive approach of communication (CCO), ventriloquism. 